\title{
Risk factors of different congenital heart defects in Guangdong, China
}

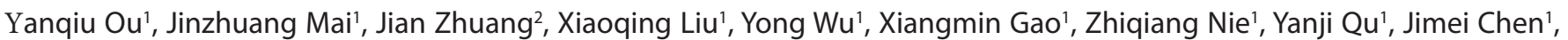
Christine Kielb3 ${ }^{3}$ Ursula Lauper ${ }^{3}$ and Shao Lin ${ }^{3}$

Pediatric Research 79: 549-558; advance online publication, January 20, 2016; doi:10.1038/pr.2015.264

In the originally published version of this article, the author affiliations were incorrect. The correct affiliations appear below: ${ }^{1}$ Department of Cardiovascular Disease Epidemiology, Guangdong Cardiovascular Institute, Guangdong Provincial Key Laboratory of South China Structural Heart Disease, Guangdong General Hospital (Guangdong Academy of Medical Sciences), Guangzhou, Guangdong, China; ${ }^{2}$ Department of Cardiac Surgery, Guangdong Cardiovascular Institute, Guangdong Provincial Key Laboratory of South China Structural Heart Disease, Guangdong General Hospital (Guangdong Academy of Medical Sciences), Guangzhou, Guangdong, China; ${ }^{3}$ Bureau of Environmental and Occupational Epidemiology, Center for Environmental Health, New York State Department of Health, Albany, New York.

The authors regret the error. 